\title{
ON THE FORCE BETWEEN ROTATING COAXIAL BLACK HOLES
}

\author{
GILBERT WEINSTEIN
}

\begin{abstract}
We study the force between rotating coaxial black holes, as it was defined in [9 and 10]. We show that under a certain limit, the force is attractive, and in fact tends to infinity. This lends support to the conjecture that the force is always positive.
\end{abstract}

\section{INTRODUCTION}

The Einstein vacuum equations reduce in the stationary axially symmetric case to an axially symmetric harmonic map from $\mathbb{R}^{3}$ into the hyperbolic plane with prescribed blow-up on the axis and at infinity. This problem is used to model rotating coaxial black holes in equilibrium. The number of black holes is imposed by the choice of singular boundary conditions, see [9]. For one black hole, an explicit solution was discovered by Kerr [4], and proven to be unique by Robinson [8], based on work of Carter [2], see also [3].

The existence, regularity, and uniqueness of solutions of the reduced problem with two black holes has been established $[9,10]$. However, when reconstructing the spacetime metric from a solution of the reduced equations, one additional regularity condition is to be checked: the absence of conical singularities on the axis of symmetry. This condition is easily verified on the two unbounded components of the axis. The value of the deficiency angle on the bounded component can be related to the gravitational force, see [1 and 9, §5]. Although it has been conjectured for some time that this force is always attractive [7], few results are known so far. These include the small angular momentum case, which is obtained by a continuity argument [5], and the case where the problem allows an involutive symmetry, see [6].

In this paper, we prove that the force is attractive, and in fact tends to infinity, in a limiting case, when one of the black holes becomes extreme, and the distance to one of the adjacent black holes then tends to zero. Phrased loosely, this says that rapidly rotating black holes always attract at close range. This result lends support to the conjecture that the force is always attractive, and implies a nonexistence result for the Einstein vacuum equations. The proof is an application of an a priori bound which was essentially obtained in the existence proof. We state all the results - the existence, uniqueness, and regularity, obtained in [10], and the new results-for any number $N$ of black holes.

Received by the editors March 10, 1993.

1991 Mathematics Subject Classification. Primary 83C57.

Key words and phrases. Black holes, Einstein's vacuum equations, stationary, rotation.

Part of this work was completed while the author was visiting the Institute for Advanced Study. The author wishes to thank the Institute for its hospitality. 


\section{N-BLACK HOLE STATIONARY AXISYMMETRIC SPACETIMES}

We summarize the notation and previous results. We present the $N$-black hole case. The proofs of the results in this section are the same as for the case $N=2$ which was treated in $[9,10]$.

To begin, we pick $2 N$ points $p_{j} \in \mathbb{A}$, the $z$-axis in $\mathbb{R}^{3}$, and consider the $N$ intervals $I_{j} \subset \mathbb{A}$ from $p_{2 j-1}$ to $p_{2 j}$. We define

$$
\Gamma=\mathbb{A} \backslash \bigcup_{j=1}^{N} I_{j}=\bigcup_{j=1}^{N+1} \Gamma_{j},
$$

where $\Gamma_{j}$ are the connected components of $\Gamma$. We let $h_{j}$ be the potential of a uniform charge distribution on $\Gamma_{j}$, i.e.,

$$
h_{j}= \begin{cases}\log \left(r_{2 j-2}+z_{2 j-2}-z\right)-\log \left(r_{2 j-1}+z_{2 j-1}-z\right) & \text { if } 2 \leq j \leq N \\ \log \left(r_{1}-z_{1}+z\right) & \text { if } j=1, \\ \log \left(r_{2 N}+z_{2 N}-z\right) & \text { if } j=N+1\end{cases}
$$

where $r_{j}$ is the distance to $p_{j}$, and $z_{j}$ the $z$-coordinate of $p_{j}$. Here and throughout, $(\rho, z, \phi)$ will denote cylindrical coordinates on $\mathbb{R}^{3}$. We let $h=$ $\sum_{j=1}^{N+1} h_{j}$, and for future reference, we also write

$$
h=2 \log \rho+u=2 \log \rho+\sum_{j=1}^{N} u_{j},
$$

where $u_{j}$ is the potential of a uniform charge distribution on $I_{j}$. We define the norms:

$$
\|x\|_{1}=\left(\int_{\mathbb{R}^{3}}|\nabla x|^{2}\right)^{1 / 2}, \quad\|y\|_{1, h}=\left(\int_{\mathbb{R}^{3}} e^{-2 h}|\nabla y|^{2}\right)^{1 / 2},
$$

and let $H_{1,0}\left(\mathbb{R}^{3}\right)$ and $H_{1, h, 0}\left(\mathbb{R}^{3}\right)$ be the closure of $C_{0}^{\infty}\left(\mathbb{R}^{3}\right)$ under the norm $\|\cdot\|_{1}$, and $C_{0}^{\infty}\left(\mathbb{R}^{3} \backslash \Gamma\right)$ under the norm $\|\cdot\|_{1, h}$, respectively. We also use the norms $\|\cdot\|_{\infty}$, and $\|y\|_{\infty, h}=\left\|e^{-h} y\right\|_{\infty}$, and the corresponding spaces, $L^{\infty}\left(\mathbb{R}^{3}\right)$ and $L_{h}^{\infty}\left(\mathbb{R}^{3}\right)$. We pick a function $Y_{0} \in C^{\infty}$ with $\left\|Y_{0}\right\|_{1, h}<\infty$, and $\left.Y_{0}\right|_{\Gamma_{j}}=c_{j}$. Finally, we let $\mathbb{H}^{2}$ be the right half-plane model for the hyperbolic plane $\{(X, Y): X>0\}$ with its metric $\left(d X^{2}+d Y^{2}\right) / X^{2}$.

The Einstein vacuum equations for a stationary, axially symmetric, asymptotically flat spacetime reduces to the problem of finding a harmonic map: $(X, Y): \mathbb{R}^{3} \backslash \Gamma \rightarrow \mathbb{H}^{2}$, with the same asymptotic behavior as the map $\left(e^{h}, Y_{0}\right)$, near $\Gamma$, and as $r \rightarrow \infty$. Conversely, suppose a solution of the following nonlinear problem:

$$
\begin{gathered}
\Delta \log X=-|\nabla Y|^{2} / X^{2}, \\
\operatorname{div}\left(\nabla Y / X^{2}\right)=0, \\
x=\log X-h \in H_{1,0} \cap L^{\infty}, \quad x \rightarrow 0 \quad \text { as } r \rightarrow \infty, \\
y=Y-Y_{0} \in H_{1, h, 0} \cap L_{h}^{\infty}, \quad e^{-h} y \rightarrow 0 \quad \text { as } r \rightarrow \infty,
\end{gathered}
$$


is found. Then, a Ricci flat metric,

$$
d s^{2}=-V d t^{2}+2 W d t d \phi+X d \phi^{2}+e^{2 \lambda}\left(d \rho^{2}+d z^{2}\right),
$$

can be constructed on $\mathbb{R} \times\left(\mathbb{R}^{3} \backslash \mathbb{A}\right)$. To obtain the metric components, we integrate

$$
\begin{aligned}
& d \Omega=-\frac{\rho * d Y}{X^{2}}, \\
& d \beta=\frac{\rho}{2} \operatorname{Re}\left[\left((\partial u+\partial x)^{2}+\frac{(\partial Y)^{2}}{X^{2}}\right) d \zeta\right],
\end{aligned}
$$

with $\Omega, \beta \rightarrow 0$, as $r \rightarrow \infty$. Here, we have used the Hodge dual operator given by $* d \rho=d z$, and $* d z=-d \rho$, the operator $\partial=\partial_{\rho}-i \partial_{z}$, and the 1 -form $d \zeta=d \rho+i d z$. The 1 -forms on the right-hand side in (2.7) and (2.8) are closed as a consequence of the harmonic map equations for $(X, Y)$. We now take $2 \lambda=\beta+u+x, W=X \Omega$, and $V=X^{-1}\left(\rho^{2}-W^{2}\right)$. In view of the regularity result, see Theorem $2.1(\mathrm{~b})$, it follows from (2.7) and (2.8) that $\Omega$ is constant on each $I_{j}$, and $\beta$ is constant on each $\Gamma_{j}$. We define $\omega_{j}=-\left.\Omega\right|_{I_{j}}$, and $b_{j}=\left.\beta\right|_{\Gamma_{j}}$. We note that the problem is invariant under translation along the $z$-axis in $\mathbb{R}^{3}$, translation along the $Y$-axis in $\mathbb{H}^{2}$, and under the one-parameter scaling transformation given by

$$
\begin{array}{cl}
X \rightarrow a^{2} X, & Y \rightarrow a^{2} Y, \\
\rho \rightarrow a \rho, & z \rightarrow a z .
\end{array}
$$

In the case $N=1$, the solution can be written explicitly. To write down the solution, we introduce the parameters $m=\left(z_{2}-z_{1}\right) / 2$, and $L=\left(c_{2}-c_{1}\right) / 8$, and change over to the parameters $M$ and $a$ defined by $m^{2}+a^{2}=M^{2}$, and $L=M a$. Here $M$ is the total mass, and $L$ the total angular momentum. We define elliptical coordinates $(s, \theta)$, with $s>M+m$, by

$$
\rho^{2}=(s-M+m)(s-M-m) \sin ^{2} \theta, \quad z=(s-M) \cos \theta .
$$

The Kerr solution is now given by

$$
\begin{aligned}
& X=\left(s^{2}+a^{2}+\frac{2 M a^{2} s \sin ^{2} \theta}{s^{2}+a^{2} \cos ^{2} \theta}\right) \sin ^{2} \theta, \\
& Y=2 M a \cos \theta\left(3-\cos ^{2} \theta+\frac{a^{2} \sin ^{4} \theta}{s^{2}+a^{2} \cos ^{2} \theta}\right) .
\end{aligned}
$$

We note that here $h=2 \log [(s-M+m) \sin \theta]$, and that $x \in L^{\infty}\left(\mathbb{R}^{3}\right)$. In fact,

$$
1 \leq e^{x} \leq 1+a^{2} / m^{2} \text {. }
$$

Furthermore, we note that $a^{2}<M^{2}$, i.e., $|L|<M^{2}$, and $M^{2} \rightarrow|L|$ when $m \rightarrow 0$. In fact, a Kerr solution can also be written for $a^{2} \geq M^{2}$, but for $a^{2}>M^{2}$ it contains a naked singularity. When $a^{2}=M^{2}$, the solution is called extreme. We will continue to call the limit $m_{j} \rightarrow 0$ the extreme limit when $N>1$.

Given a choice of parameters for general $N$ as above, we associate a corresponding Kerr solution: $(\Phi, \Psi): \mathbb{R}^{3} \backslash\left(\Gamma_{1} \cup \Gamma_{N+1}\right) \rightarrow \mathbb{H}^{2}$, with $\left.\Psi\right|_{\Gamma_{j}}=c_{j}$ for 
$j=1$ and $j=N+1$. We write $\log \Phi=g+\varphi$, where $g=h_{1}+h_{N+1}=h-f$, and $f=\sum_{j=2}^{N} h_{j}$.

The following theorem was essentially obtained in [10]. Although it was stated and proved only for the case $N=2$, the same arguments work for any number of black holes.

Theorem 2.1. For any choice of the parameters, we have:

(a) There exists a unique solution of (2.2)-(2.5).

(b) For this solution, we have

$$
e^{\varphi-x}+e^{2 f+x-\varphi}+e^{-2 g-\varphi-x}(Y-\Psi)^{2} \leq 2,
$$

where $\left(e^{g+\varphi}, \Psi\right)$ is the corresponding Kerr solution. Furthermore $x, Y \in$ $C^{\infty}\left(\mathbb{R}^{3} \backslash\left\{p_{1}, \ldots, p_{2 N}\right\}\right) \cap C^{1}\left(\mathbb{R}^{3}\right)$, and there is a constant $C$ depending only on the parameters such that

$$
0 \leq x \leq C,
$$

and

$$
|\nabla x|^{2}+|\nabla Y|^{2} / X^{2} \leq C .
$$

(c) The metric (2.6) is asymptotically flat.

(d) For $j=1, \ldots, N+1$, we have

$$
b_{j+1}-b_{j}=-2\left(\sum_{\substack{1 \leq k \leq N \\ k \neq j}}\left\{u_{k}\left(p_{2 j}\right)-u_{k}\left(p_{2 j-1}\right)\right\}+x\left(p_{2 j}\right)-x\left(p_{2 j-1}\right)\right) \text {. }
$$

In the spacetime defined by (2.6), the intervals $I_{j}$ are actually surfaces, and it is not difficult to check that, provided the spacetime is regular, these surfaces are event horizons, see [9]. There are several physically significant quantities arising as periods of closed 1 -forms, and we shall discuss these now. If $K$ is any Killing field on a vacuum spacetime, the quantity

$$
Q_{K}(S)=\int_{S} * d K
$$

depends only on the homology class of $S$, as can easily be seen from Stokes' Theorem. For example if $K=\partial / \partial \phi$, then $L(S)=(1 / 16 \pi) Q_{K}(S)$ is the angular momentum contained in $S$, and if $K=\partial / \partial t$, then $M(S)=(1 / 8 \pi) Q_{K}(S)$ is the mass contained in $S$. Here, the second homology is generated by surfaces $S_{j}$, where $S_{j}$ is any surface containing only the $j$ th black hole, and we set $M_{j}=M\left(S_{j}\right)$, and $L_{j}=L\left(S_{j}\right)$, the mass and angular momentum of the $j$ th black hole respectively. Strictly speaking, we have a vacuum spacetime only outside $\Gamma$, but it is not difficult to check that there is no contribution to (2.16) from integration along $\Gamma$. Possibly deforming $S_{j}$ through a homotopy, we may take $S_{j}$ axially symmetric, and we obtain

$$
\begin{aligned}
M_{j} & =\frac{1}{4} \int_{C_{j}} * \frac{X d V+W d W}{\rho}, \\
L_{j} & =\frac{1}{8} \int_{C_{j}} * \frac{X d W-W d X}{\rho},
\end{aligned}
$$


where $C_{j}$ is any curve from $\Gamma_{j}$ to $\Gamma_{j+1}$. It follows immediately from (2.7) that $L_{j}=\left(c_{j+1}-c_{j}\right) / 8$. Furthermore, we have

$$
M_{j}=m_{j}+2 \omega_{j} L_{j},
$$

where $2 m_{j}=z_{2 j}-z_{2 j-1}$. Indeed, a straightforward calculation yields

$$
(X d V+W d W) / \rho=\Omega * d Y-\rho(d u+d x) .
$$

Equation (2.19) now follows, by letting $C_{j}$ tend to $I_{j}$, and noting that $\rho u_{\rho} \rightarrow$ -2 on $I_{j}$, see $[10, \S 6]$. The constant $\omega_{j}$ can be interpreted as the angular velocity of the $j$ th black hole. Finally, we have the periods of $d \beta$ :

$$
b_{j+1}-b_{j}=\int_{C_{j}} d \beta .
$$

Since the metric (2.6) is written in cylindrical coordinates, it extends across $\Gamma$ only if there is no conical singularity on the axis. The deficiency angle on $\Gamma_{j}$ is measured by $b_{j}$. It was shown in [9] that

$$
F_{j}=\frac{1}{4}\left(e^{-b_{j} / 2}-1\right)
$$

could be interpreted as a gravitational force.

\section{THE FORCE IN THE EXTREME LIMIT}

In this section, we prove that the force is attractive when one of the black holes is close to being extreme, and the distance to an adjacent black hole is small. We first consider the case where all the other black holes are on the same side of the one close to being extreme. This is always the case when $N=2$.

Proposition 3.1. For any solution of (2.2)-(2.5), we have

$$
\begin{aligned}
x\left(p_{2}\right)-x\left(p_{1}\right) \geq & -\log 4+2 f\left(p_{1}\right)-2 g\left(p_{2}\right)-\varphi\left(p_{1}\right)-\varphi\left(p_{2}\right) \\
& +2 \log \left|c_{2}-\Psi\left(p_{2}\right)\right| .
\end{aligned}
$$

Proof. From part (b) of Theorem 2.1, we have

$$
\begin{aligned}
& x\left(p_{1}\right) \leq \log 2-2 f\left(p_{1}\right)+\varphi\left(p_{1}\right), \\
& x\left(p_{2}\right) \geq-\log 2-2 g\left(p_{2}\right)-\varphi\left(p_{2}\right)+2 \log \left|c_{2}-\Psi\left(p_{2}\right)\right| .
\end{aligned}
$$

Summing up, we obtain (3.1).

Note that on $\Gamma_{2}-2 \sum_{k=2}^{N}\left\{u_{k}\left(p_{2}\right)-u_{k}\left(p_{2}\right)\right\}$, the linear contribution to $b_{2}$ in (2.15), is always negative. We now show that the nonlinear contribution $-2\left(x\left(p_{2}\right)-x\left(p_{1}\right)\right)$ is also negative in the extreme limit. Thus, we obtain that the force $F_{2}$, see (2.20), is positive in this case. Let $L=\sum_{j=1}^{N} L_{j}$ denote the total angular momentum.

Theorem 3.1. Fix $p_{j}$, for $1 \leq j \leq 2 N$. For any constant $C_{1}>0$, there is a constant $C_{2}$ such that if $|L| \leq C_{1}$, and $\left|L_{1}\right|>C_{2}$, then $x\left(p_{2}\right)-x\left(p_{1}\right)>0$, for any solution of $(2.2)-(2.5)$.

Proof. Suppose $|L| \leq C_{1}$, then all the terms are bounded in the estimate (3.1) except for the term

$$
2 \log \left|c_{2}-\Psi\left(p_{2}\right)\right|
$$

which tends to $\infty$ when $\left|c_{1}-c_{2}\right| \rightarrow \infty$. The theorem follows. 
This theorem can be interpreted roughly as saying that the force between rapidly counterrotating black holes is always attractive. In order to remove the counterrotation assumption, we will have to assume that the black holes are not too far apart. However, note that if one wished to keep the angular momenta bounded in Theorem 3.1, as is the case in Theorem 3.2, one would have to rescale the solution using (2.9), which would again have the effect of bringing the black holes close together. Let $2 d_{j}=z_{2 j-1}-z_{2 j-2}$ be the length of $\Gamma_{j}$, and $2 m=z_{2 N}-z_{1}$.

Theorem 3.2. Fix all the points $p_{j}$ except $p_{2}$, and $c_{j}$ for $1 \leq j \leq N+1$, such that

$$
\left|L_{1}\right|>4 D_{2}\left(m^{2}+|L|\right) /\left(D_{2}+m_{2}\right),
$$

where $D_{2}=m_{1}+d_{2}$. Then, there is a constant $\varepsilon>0$ such that if $m_{1}<\varepsilon$, we have $x\left(p_{2}\right)-x\left(p_{1}\right)>0$, for any solution of $(2.2)-(2.5)$.

Proof. Note first that in the statement of the theorem $D_{2}$ is fixed although $p_{2}$ is not. Now, it follows from (2.10) that $e^{\varphi} \leq\left(1+|L| / m^{2}\right)$. In view of $(2.1)$, we have

$$
f\left(p_{1}\right)=h_{2}\left(p_{1}\right)+\sum_{j=3}^{N} h_{j}\left(p_{1}\right) \geq \log \left(\frac{m_{1}}{d_{2}+m_{1}}\right)+\log \left(\frac{m_{1}+d_{2}+m_{2}}{m}\right) .
$$

The last inequality follows from

$$
\sum_{j=3}^{N} h_{j}\left(p_{1}\right)=\sum_{j=3}^{N} \log \left(\frac{r_{2 j-2}\left(p_{1}\right)}{r_{2 j-1}\left(p_{1}\right)}\right) \geq \log \left(\frac{r_{4}\left(p_{1}\right)}{r_{2 N}\left(p_{1}\right)}\right)=\log \left(\frac{m_{1}+d_{2}+m_{2}}{m}\right) .
$$

Furthermore, again using (2.1), we find

$$
g\left(p_{2}\right)=h_{1}\left(p_{2}\right)+h_{N+1}\left(p_{2}\right)=\log \left[16 m_{1}\left(m-m_{1}\right)\right] .
$$

Substituting in (3.1), we obtain

$$
x\left(p_{2}\right)-x\left(p_{1}\right) \geq 2 \log \left[\frac{\left(D_{2}+m_{2}\right)\left|c_{2}-\Psi\left(p_{2}\right)\right|}{32 m D_{2}\left(m-m_{1}\right)\left(1+|L| / m^{2}\right)}\right] .
$$

When $m_{1} \rightarrow 0$, we have $p_{2} \rightarrow p_{1}$, hence the right-hand side tends to

$$
2 \log \left[\frac{\left(D_{2}+m_{2}\right)\left|L_{1}\right|}{4 m^{2} D_{2}\left(1+|L| / m^{2}\right)}\right],
$$

which is positive by (3.2). The theorem follows.

Note that (3.2) can be achieved by taking $D_{2}$ small enough. Thus, this theorem can be interpreted roughly as saying that extreme black holes always attract at close range. Furthermore, one sees from the proof that in the limit $D_{2} \rightarrow 0$ the force tends to $\infty$.

We now treat the case where the extreme black hole is not the first or the last one along $\mathbb{A}$. Although the main idea is the same, we need a new estimate similar to (2.12). Let $(X, Y)$ be an $N$-black hole solution of (2.2)-(2.5), and fix $2 \leq k \leq N-1$. We will associate with this solution an $(N-1)$-black hole solution $\left(X^{\prime}, Y^{\prime}\right)$ of $(2.2)-(2.5)$. We take

$$
\Gamma^{\prime}=\Gamma \backslash \Gamma_{k+1}=\bigcup_{\substack{1 \leq j \leq N+1 \\ j \neq k+1}} \Gamma_{j}, \quad h^{\prime}=h-h_{k+1}=\sum_{\substack{1 \leq j \leq N+1 \\ j \neq k+1}} h_{j}
$$


and we use the existence theorem with $\left.Y^{\prime}\right|_{\Gamma_{j}}=c_{j}$ for $1 \leq j \leq N+1, j \neq k+1$. We set $\log X^{\prime}=h^{\prime}+x^{\prime}$, and obtain from Theorem 2.1 part (b):

$$
0 \leq x^{\prime} \leq C^{\prime}
$$

where $C^{\prime}$ is independent of $p_{2 k}, p_{2 k+1}$, and $c_{k+1}$, see (2.13).

Proposition 3.2. For the solutions $(X, Y)$ and $\left(X^{\prime}, Y^{\prime}\right)$ above, we have

$$
e^{x^{\prime}-x}+e^{2 h_{k+1}+x-x^{\prime}}+e^{-2 h^{\prime}-x^{\prime}-x}\left(Y-Y^{\prime}\right)^{2} \leq 2 .
$$

Proof. The proof is similar to the one used in [10] to obtain (2.12). Let $d=$ $\operatorname{dist}\left[\left(X^{\prime}, Y^{\prime}\right),(X, Y)\right]$ be the distance in $\mathbb{H}^{2}$ between the two solutions. Since $\mathbb{H}^{2}$ is negatively curved, and $\left(X^{\prime}, Y^{\prime}\right)$ and $(X, Y)$ are both harmonic maps, it follows that $d^{2}$ is subharmonic in $\mathbb{R}^{3} \backslash \Gamma$. Let $\delta=\log \cosh d+h_{k+1}$, then since $\log \cosh d$ is a convex function of $d^{2}, \Delta \delta \geq 0$ in $\mathbb{R}^{3} \backslash \Gamma$. A straightforward calculation yields

$$
\delta=\log \frac{1}{2}\left[e^{x^{\prime}-x}+e^{2 h_{k+1}+x-x^{\prime}}+e^{-2 h^{\prime}-x^{\prime}-x}\left(Y-Y^{\prime}\right)^{2}\right] .
$$

Using Lemma 1 in [10], we find that $\delta \in H_{1, \text { loc }}\left(\mathbb{R}^{3}\right)$, and therefore $\Delta \delta \geq 0$ weakly in all of $\mathbb{R}^{3}$. Since $\delta \rightarrow 0$ as $r \rightarrow \infty$, the maximum principle gives $\delta \leq 0$, and the proposition follows.

We now prove

Theorem 3.3. Fix $p_{j}$ for $1 \leq j \leq 2 N, j \neq 2 k$, and $c_{j}$ for $1 \leq j \leq N+1$, such that

$$
d_{k}\left|L_{k}\right|>e^{C^{\prime}} m^{2} D_{k+1},
$$

where $D_{k}=m_{k}+d_{k+1}$, and with $C^{\prime}$ as in (3.3). Then, there is a constant $\varepsilon>0$ such that if $m_{k}<\varepsilon$, we have $x\left(p_{2 k}\right)-x\left(p_{2 k-1}\right)>0$, for any solution of (2.2)-(2.5)

Proof. As before $D_{k}$ is fixed although $p_{2 k}$ is not. From (3.4), we have

$$
\begin{aligned}
x\left(p_{2 k-1}\right) & \leq \log 2-2 h_{k+1}\left(p_{2 k-1}\right)+x^{\prime}\left(p_{2 k-1}\right), \\
x\left(p_{2 k}\right) & \geq-\log 2-2 h^{\prime}\left(p_{2 k}\right)-x^{\prime}\left(p_{2 k}\right)+2 \log \left|c_{k+1}-Y^{\prime}\left(p_{2 k+1}\right)\right| .
\end{aligned}
$$

Summing up, and using (3.3), we obtain

$$
\begin{aligned}
x\left(p_{2 k}\right)-x\left(p_{2 k-1}\right) \geq & -\log 4+2 h_{k+1}\left(p_{2 k-1}\right)-2 h^{\prime}\left(p_{2 k}\right) \\
& -2 C^{\prime}+2 \log \left|c_{k+1}-Y^{\prime}\left(p_{2 k}\right)\right| .
\end{aligned}
$$

Now, using (2.1), we have

$$
h_{k+1}\left(p_{2 k-1}\right)=\log \left(m_{k} / D_{k+1}\right)
$$

and, since $h_{j} \leq 0$ for $2 \leq j \leq N$, we find

$$
h^{\prime}\left(p_{2 k}\right) \leq g\left(p_{2 k}\right)+h_{k}\left(p_{2 k}\right) \leq \log \left(4 m^{2} m_{k} /\left(m_{k}+d_{k}\right)\right) .
$$

The last inequality follows since $g$ has its maximum $\log 4 m^{2}$ on $\mathbb{A}$ at the midpoint between $p_{1}$ and $p_{2 N}$. Substituting (3.6) and (3.7) into (3.5), we obtain

$$
x\left(p_{2 k}\right)-x\left(p_{2 k-1}\right) \geq 2 \log \left[\frac{\left(m_{k}+d_{k}\right)\left|c_{k+1}-Y^{\prime}\left(p_{2 k}\right)\right|}{8 e^{C^{\prime} m^{2} D_{k+1}}}\right] .
$$


When $m_{k} \rightarrow 0, p_{2 k} \rightarrow p_{2 k-1}$, and the right-hand side above tends to

$$
2 \log \left[\left|L_{k}\right| d_{k} / e^{C^{\prime}} m^{2} D_{k+1}\right],
$$

which is positive by (3.4). The theorem follows.

Note that again (3.4) can be achieved by taking $D_{k+1}$ small enough. Also note that when $D_{k+1}$ is small, the linear contribution to $b_{k+1}$ is negative too, so that the force $F_{k+1}$ is positive. This implies a nonexistence result for the Einstein vacuum equations, see [9].

Corollary 3.1. Fix $m_{j}$ and $d_{j+1}$ for $1 \leq j \leq N, j \neq k$, and $L_{j}$ for $1 \leq j \leq N$, with $L_{k} \neq 0$. Then there are constants $\varepsilon_{1}>\varepsilon_{2}>0$ such that if $m_{k}+d_{k+1}<\varepsilon_{1}$, and $m_{k}<\varepsilon_{2}$, we have for any solution of (2.2)-(2.5) $F_{k+1}>0$. Consequently, there is no asymptotically flat, stationary, axially symmetric solution of the Einstein vacuum equations corresponding to these parameter values.

\section{REFERENCES}

1. R. Bach and H. Weyl, Neue Lösungen der Einsteinschen Gravitationsgleichungen, Math. Z. 13 (1921), 132-145.

2. B. Carter, Black hole equilibrium states, in Black Holes (C. DeWitt and B. S. DeWitt, eds.), Gordon and Breach, New York, 1973.

3. __ Bunting identity and Mazur identity for nonlinear systems including the black hole equilibrium system, Comm. Math. Phys. 99 (1985), 563-591.

4. S. W. Hawking and G. F. R. Ellis, The large scale structure of space-time, Cambridge Univ. Press, Cambridge, 1973.

5. Y. Li and G. Tian, Regularity of harmonic maps with prescribed asymptotic behavior and applications, Comm. Math. Phys. 149 (1992), 1-30.

6. __ Nonexistence of axially symmetric, stationary solution of Einstein vacuum equation with disconnected symmetric event horizon, Manuscripta Math. 73 (1991), 83-89.

7. R. Penrose, Some unsolved problems in classical general relativity, in Seminar on Differential Geometry (S. T. Yau, ed.), Ann. of Math. Studies, no. 102, Princeton Univ. Press, Princeton, N.J., 1982.

8. D. C. Robinson, Uniqueness of the Kerr black hole, Phys. Rev. Lett. 34 (1975), 905-906.

9. G. Weinstein, On rotating black holes in equilibrium in general relativity, Comm. Pure Appl. Math. 43 (1990), 903-948.

10. _ The stationary axisymmetric two-body problem in general relativity, Comm. Pure Appl. Math. 45 (1992), 1183-1203.

Department of Mathematics, University of Alabama at Birmingham, Birmingham, AlABAMA 35294-0001

E-mail address: weinstei@math.uab.edu 